\title{
ORIENTACIÓN AL MERCADO EN HOSPITALES Y CLÍNICAS DE LOS ESTADOS DE AGUASCALIENTES Y TABASCO
}

\author{
Miguel Ángel Ramírez Martínez*, Clara Luz Lamoyi Bocanegra**, Lenin Martínez Pérez ${ }^{* * *}$.
}

\begin{abstract}
Ramírez-Martínez M.A., Lamoyi-Bocanegra C.L., Martínez-Pérez $L$. Orientación al mercado en hospitales y clinicas de los Estados de Aguascalientes y Tabasco. Hitos de Ciencias Económico Administrativas 2013;19 (54): 55-66.
\end{abstract}

\section{RESUMEN}

Objetivo: Mostrar el grado de Orientación al Mercado (OM) que tienen las empresas (hospitales y clínicas) de los Estados de Aguascalientes y Tabasco, utilizando el Modelo de Orientación al Mercado, desarrollado por Kohli y Jaworski (1990) y Narver y Slater (1990) y que fueron adaptados a México por Martínez Serna y Pinzón (2009).

Material y método: Se aplicó un cuestionario a los propietarios y/o gerentes de los establecimientos, este utiliza la escala de Likert; los resultados son evaluados con el paquete SPSS 15, obteniendo las medias de cada una de las variables evaluadas para establecer el grado de orientación al mercado.

Resultados: La investigación comprueba que el modelo puede ser aplicado a las empresas medianas y grandes de esta rama del sector servicios de la economía mexicana.

Conclusiones: La mayor parte de las empresas del sector servicio (hospitales y clínicas) de los dos Estados, tienen un alto grado de Orientación al Mercado, aunque el Estado de Aguascalientes cuenta con un mayor número de empresas con un grado de OM bajo, por lo anterior se elaboran una serie de estrategias que al ser aplicadas podrían mejorar el grado de OM de las empresas que se encuentran en el nivel bajo e incrementar la competitividad.
Ramírez-Martínez M.A., Lamoyi-Bocanegra C.L., Martínez-Pérez $L$. Market orientation in hospitals and clinics of Aguascalientes and Tabasco. Hitos de Ciencias Económico Administrativas 2013;19 (54): 55-66.

\section{ABSTRACT}

Objective: Research shows the degree of market orientation (MO) that the companies (hospitals and clinics) have in the states of Aguascalientes and Tabasco by using the model of market orientation developed by Kohli and Jaworski (1990), and Narver and Slater (1990), which was adapted to Mexico by Martinez Serna and Pinzon (2009).

Material and method: A questionnaire that uses the Likert scale is applied to the owners and/or managers of establishments; the results are evaluated with the SPSS 15 package to calculate the mean of each of the evaluated variables so that the degree of market orientation is determined.

Results: The research demonstrates that the model can be applied to medium and large companies of this branch of the service sector of the Mexican economy.

Conclusions: Most of the companies in the service sector (hospitals and clinics) of both states have a high degree of market orientation, although the state of Aguascalientes has a greater number of companies with a low MO degree, that is why a series of strategies are developed so that they could be applied in order to improve the degree of $\mathrm{MO}$ of businesses that are in the low level and increase their competitiveness.

Palabrasclaves: Orientaciónalmercado. Aguascalientes.

Key words: Market orientation. Aguascalientes. Tabasco. Hospitales y clínicas.

Tabasco. Hospitals and Clinics.

DIRECCIÓN PARA RECIBIR CORRESPONDENCIA: Correo electrónico: mangel113@hotmail.com

*Doctor en Antropología Social. Profesor-Investigador. División Académica de Ciencias Económico Administrativas. Universidad Juárez Autónoma de Tabasco.

** Doctora en Administración. Profesor-Investigador. División Académica de Ciencias Económico Administrativas. Universidad Juárez Autónoma de Tabasco.

${ }^{* * *}$ Maestro en Finanzas. Profesor-Investigador. División Académica de Ciencias Económico Administrativas. Universidad Juárez Autónoma de Tabasco. 
a falta de estudios comparativos en México entre las empresas que integran las mismas actividades económicas en cada uno de Estados del país, nos llevó a la realización de una investigación cuya finalidad es conocer el grado de Orientación a Mercado que tienen los hospitales y clínicas privadas del municipio del Centro (Villahermosa) del Estado de Tabasco ${ }^{1}$ y de Aguascalientes ${ }^{2}$, lo que permitirá identificar la competitividad de dicho sector en cada uno de los Estados y posteriormente, proponer una serie de recomendaciones (estrategias) que al ser aplicadas por el empresario incrementarían el grado de OM de su empresa y del sector en general, a través de ello se busca la maximización de los beneficios y su permanencia en los mercados globalizados.

El sector terciario de la economía ha crecido a pasos gigantescos en los últimos años. A nivel mundial, el desarrollo de diferentes empresas que brindan paralelamente los mismos servicios, ha hecho muy notable la competencia entre ellas, por lo que se ha intensificado la puesta en práctica de la tecnología del marketing para lograr diferenciación en el mercado, formar parte de la elección de los clientes y obtener sus objetivos de lucro individuales. En los últimos años se han desarrollado nuevos enfoques y nuevos modelos dentro del marketing, los cuales se han aplicado en economías principalmente desarrolladas (Unión Europea, Estados Unidos y Canadá) dando como resultados que las empresas alcancen mayores niveles de competitividad en las economías globalizadas.

En México, son pocos los avances en el desarrollo de modelos de marketing y se desconoce que hacen las empresas en términos de marketing para enfrentar a las empresas globales dentro del territorio nacional, por tal motivo, el estudio utilizó el enfoque de Orientación a Mercado (OM) al demostrarse, a través de investigaciones realizadas en la Unión Americana

\footnotetext{
${ }_{1}^{1}$ Tabasco tiene una superficie de $24578 \mathrm{~km}^{2}$, lo que representa el $1,3 \%$ de la superficie nacional. Según el INEGI en el 2005 cuenta con una población de 2, 056,911, en 17 municipios. El municipio de Centro cuenta con 597,880 habitantes, lo que representa el $29.1 \%$ de la población total del Estado.

${ }^{2}$ Aguascalientes tiene una extensión territorial de $5.471 \mathrm{~km}^{2}$, lo que representa el $0,03 \%$ de la superficie total de México. Según el INEGI en el 2005 cuenta con una población de 1, 065,416. Cuenta en 11 municipios. El municipio de Aguascalientes cuenta con 723,043 habitantes, lo que representa el $67.9 \%$ de la población total del Estado.
}

y en España, principalmente, que éste puede generar ventajas competitivas y con ello el éxito de las empresas. Finalmente, es del interés del investigador el corroborar si este tipo de modelos explican el comportamiento de las empresas localizadas en ciudades medianas mexicanas.

\section{MARCO TEÓRICO}

\section{MODELOS DE ORIENTACIÓN A MERCADO}

Al hacer una revisión literaria sobre los trabajos realizados con el enfoque de Orientación a Mercados, todos los autores señalan a los modelos de Kohli y Jaworski (1990) y Narver y Slater (1990) como los iniciadores del enfoque de Orientación a Mercados. Los primeros consideran la orientación a mercado como procesos de inteligencia de mercados, la cual se integra de: i) la generación de información proveniente del mercado, ii) la forma como se disemina en la organización y iii) la respuesta de la organización ante dicha información. Los segundos consideran la orientación al mercado como cultura organizacional a través de la cual se genera comportamientos que responden a las necesidades del mercado y al mismo tiempo se genera un crecimiento interno permanente. Martínez (2004, p.38) señala que en estudios recientes la Orientación al Mercado es una cultura organizacional; en otros se le ha considerado como una filosofía, y en algunos otros como un conjunto de comportamientos, los cuales de manera más eficiente y efectiva crean superior valor al cliente y superior desempeño para la organización.

\section{MEDICIÓN DEL GRADO DE ORIENTACIÓN AL} MERCADO: DISTINTAS ESCALAS

La medición de la Orientación al Mercado es el paso siguiente al establecimiento de la definición operativa, se lleva a cabo por medio de escalas, siendo tres las más importantes: Escala MKTOR de Narver y Slater (1990), Escala MARKOR de Kohli, Jaworski y Kumar (1993), y la Escala de Lambin (1995). La primera, consta de 15 ítems, que miden los tres componentes de la Orientación al Mercado recogidos en su definición de orientación al mercado (i.e.: orientación al cliente, orientación a la competencia e interdependencia funcional) y otros nueve ítems sobre resultados para medir primeramente el grado de orientación y posteriormente, su relación con los resultados de la empresa. Pinzón y Serna (2009) señalan que Narver y Slater entienden que la $\mathrm{OM}$ es un constructo unidimensional en el que se integran tres componentes comportamentales y dos 
criterios de decisión, aunque estos dos últimos no fueron corroborados en la contrastación empírica. La segunda, se integra de 20 ítems en su versión reducida que miden los tres componentes de la orientación al mercado ya mencionados y usan en su combinación otra de cinco ítems para medir la relación del grado de orientación con los resultados. La tercera, considera nueve componentes: análisis de clientes, acciones estratégicas sobre los clientes, análisis de clientes intermedios, acciones estratégicas sobre clientes intermedios, análisis de la competencia, acciones estratégicas sobre la competencia, análisis del entorno, acciones estratégicas sobre el entorno y coordinación interfuncional.

\section{MODELO DE ORIENTACIÓN AL MERCADO UTILIZADO EN EL ESTUDIO}

El modelo (Figura 1) que se utiliza incluye aspectos para medir las variables conflicto y conexión interdepartamental que corresponden a la dinámica interdepartamental, así como la formalización, centralización y orientación del sistema de recompensas que son parte del sistema organizacional; también, compromiso organizacional, espíritu de equipo y desempeño total que forman parte del modelo desarrollado por Kohli y Jaworski en 1993 y para medir el constructo de Orientación a Mercado utilizaron la escala diseñada por Lambin en 1995 con las adecuaciones hechas por Rivera y Lado en 1998.

\section{ESTADO DEL ARTE}

En los últimos 20 años se ha desarrollado a nivel mundial un modelo de marketing que propone un enfoque de Orientación al Mercado (OM), el cual tiene como objetivo que las empresas conozcan el grado de OM que tienen y de esta manera poder reorientar la estrategia que les permita alcanzar la competitividad en los mercados globales.

Al hacer un análisis de las investigaciones que se realizan con el enfoque OM, se encontraron a nivel internacional: Bigné E, Küster I, Andreu Blesa y Andreu L. (2001); Iguaran R, Chávez E y Pérez J. (2006); Lonchi i Andreu Joan L. (1993); Lonchi i Andreu, J, López Belbeze Ma., y Eusebio R (1999); Olavarrieta S, Friedmann R, Hidalgo C. (1999) Riveros S, Jorge y Berné C. (2006) Santos Vijande Ma.; Vázquez Casielles R y Álvarez González L (2002); Valenzuela Fernández L, García de Madagariaga J y Blasco López Ma. (2006); Valenzuela Fernández L, García de Madagariaga J y

\section{FIGURA 1. CASA DE LA CALIDAD CON SUS 6 PARTES}

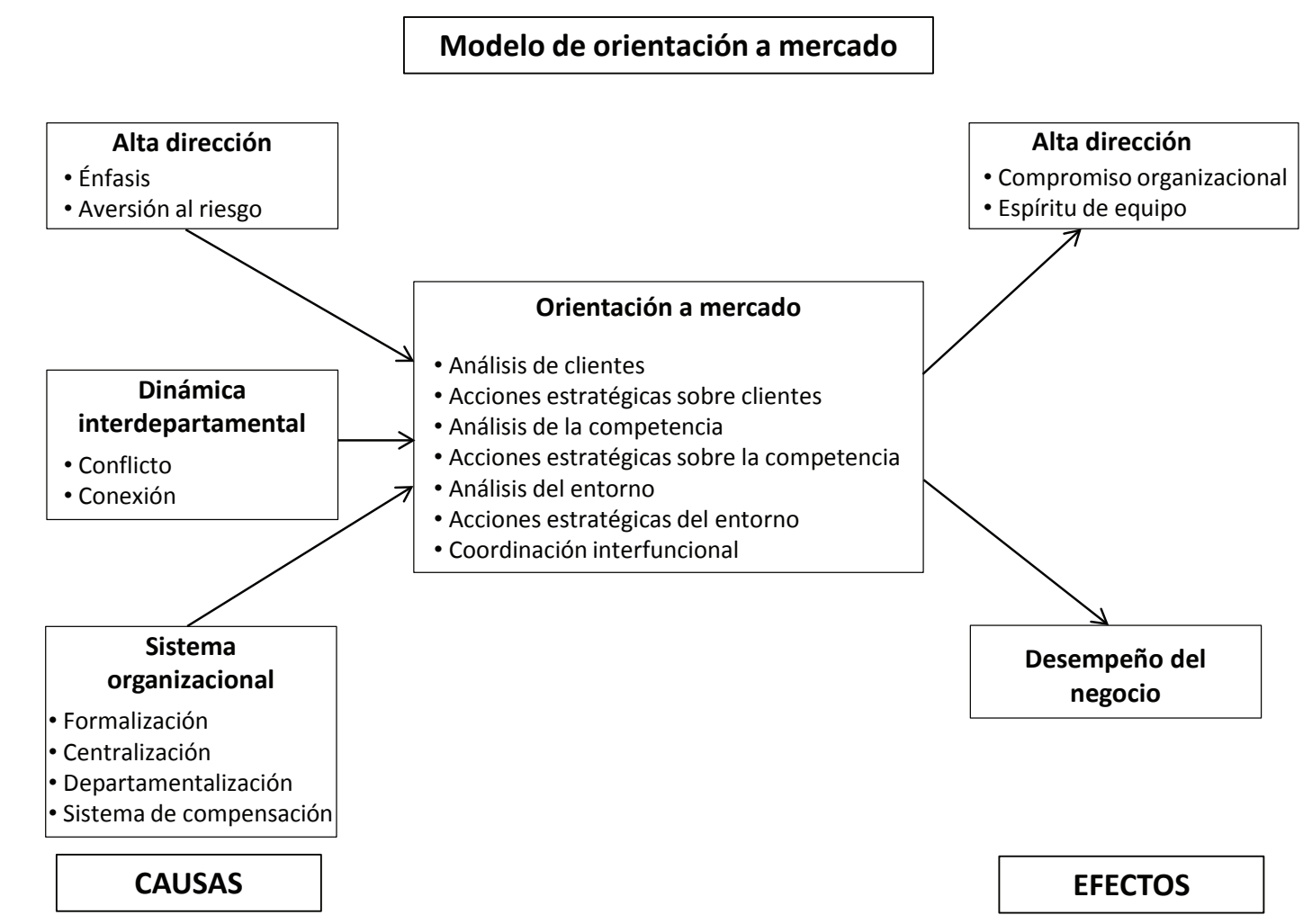

Fuente: Adaptación de Pinzón y Martínez Serna (2009) del modelo de Kohli y Jaworski (1993). 
Blasco López Ma (2007), quienes lo aplican a estudios de sectores o empresas industriales (química) y de servicios (cerámica, hoteles y hospitales).

En cuanto a los estudios en México, Serna (2004:31) señala que en la tesis: La Orientación al Mercado de las empresas en México: causas y consecuencias, de Rogerio Domenge y Luz Aleidan Martínez, evalúan empíricamente el comportamiento de las organizaciones en cuanto a su grado de OM. En el trabajo titulado: Orientación al Mercado: Un modelo desde la perspectiva de aprendizaje organizacional (2004), Martínez Serna propone un modelo de Orientación al Mercado desde una perspectiva de aprendizaje organizacional. Pinzón (2009) en su tesis doctoral, titulada: «Impacto de la Orientación al Mercado en la innovación en empresas de Aguascalientes» establece un modelo en base al estudio empírico que permite evaluar el impacto de la Orientación al Mercado en la innovación en empresas de Aguascalientes y evaluar empíricamente las relaciones entre antecedentes (causas), Orientación al Mercado e innovación en dichas empresas.

\section{MATERIAL Y MÉTODO}

Se utilizó el diseño metodológico que manejaron Pinzón y Serra (2009) en el trabajo presentado con el título de «la Orientación al Mercado (OM) y su influencia en el desempeño de hoteles y hospitales de Aguascalientes», señalando que se trataba de un estudio tipo descriptivo y correlacional. Se utilizó el cuestionario que fue proporcionado por las investigadoras que se integra de 82 reactivos y se utiliza como base la escala desarrollada por Lambin en 1995, que contempla nueve componentes: Análisis de Clientes, Acciones Estratégicas sobre Clientes, Análisis de Clientes Intermedios, Acciones Estratégicas sobre Clientes Intermedios, Análisis de la Competencia, Acciones Estratégicas sobre la Competencia, Análisis del Entorno, Acciones Estratégicas sobre el Entorno y Coordinación Interfuncional. Dichas autoras señalan: En virtud de la complejidad de la escala, Lado, Maydeu y Rivera (1998) y Lado, Maydeu y Martínez (1998), simplificaron la escala quedando de 41 ítems que evalúan los siguientes componentes: Análisis de Clientes, Acciones Estratégicas sobre Clientes, Análisis de la Competencia, Acciones Estratégicas sobre la Competencia, Análisis del Entorno, Acciones Estratégicas sobre el Entorno y Coordinación Interfuncional. Así pues, estos 41 ítems nos dan el Índice de Orientación al Mercado de las unidades de negocios. Además de medir el Índice de Orientación al Mercado con las dimensiones antes mencionadas, las autoras incluyeron en el cuestionario otras dimensiones utilizadas por Kohli y Jaworski (1993) que son consideradas antecedentes de la Orientación al Mercado y que son Conflicto Interdepartamental y Conexión Interdepartamental, (que conforman la Dinámica Interdepartamental), Formalización, Centralización y Orientación del Sistema de Recompensas (que pertenecen a los Sistemas Organizacionales), además de las dimensiones Compromiso Organizacional y Espíritu de Equipo (que son efectos sobre los empleados) y Desempeño Total también evaluadas por Kohli, Jaworski y Kumar, que son consideradas efectos de la Orientación al Mercado.

Entre las causas -señalan- por las que eligieron la escala desarrollada por Lambin para medir el nivel de Orientación al Mercado, es que la aplicación de esta escala a partir de los trabajos de Rivera (1995) y Lado (1995) pone de manifiesto que la propuesta de Lambin no es alternativa a las escalas MARKOR y MKTOR, sino que por el contrario las contiene, siendo por tanto más general que aquellas.

\section{MÉTODOS Y PROCEDIMIENTOS}

La población objetivo de esta investigación fueron los Hospitales privados y las clínicas que se encuentran localizadas en el municipio del Centro, Tabasco. Debido a que la población total de hospitales del municipio del Centro que se integra en el directorio de la Secretaría de Salud del Estado es de 28, se decidió tomar a la población total para la investigación.

El trabajo de campo se realizó de Septiembre de 2009 a Febrero de 2011, periodo en que se entregaron y recolectaron los cuestionarios en los hospitales, lo que representó un trabajo complejo, debido a la desconfianza, de los propietarios y gerentes de las clínicas y hospitales para proporcionar los datos, por el clima de inseguridad que se presentaba en el estado. De total (28) de hospitales y clínicas a quienes se les invitó a responder el cuestionario: 23 lo contestaron y cinco empresas no accedieron a participar o no regresaron el cuestionario al momento del cierre del trabajo de campo, lo que representó un índice de respuesta del $82.1 \%$. 
En el estudio se utilizó la estadística descriptiva: la distribución de frecuencias y media; el coeficiente de correlación de Pearson y el coeficiente de Cronbach. La correlación se utilizó para determinar si existía asociación entre las variables Conflicto Interdepartamental y Orientación al Mercado; entre Conexión Interdepartamental y OM; entre Formalización y Orientación al Mercado; entre Centralización y Orientación a Mercado; entre Orientación al Sistema de Recompensas y Orientación al Mercado; entre Orientación al Mercado y Compromiso Organizacional; Orientación al Mercado y Espíritu de Equipo; y, entre Orientación al Mercado y Desempeño Total.

Se probó la validez y confiabilidad del instrumento ocupando el método de Consistencia Interna de la escala tipo Likert que midió la Orientación al Mercado, sus causas (antecedentes) y efectos, para lo cual se realizó la prueba de Alpha Cronbach ${ }^{3}$. A través de esta prueba se revisaron cada una de las dimensiones propuesta encontrándose que la subdimensión de acciones estratégicas sobre el entorno, del concepto de Orientación al Mercado, no presentó consistencia interna ${ }^{4}$ en virtud de que no supera el valor 0.60 de Alpha de Cronbach.

En cuanto a los antecedentes de la Orientación al Mercado en los hospitales y clínicas de la ciudad de Villahermosa, Tabasco, el número de ítems que integraban el concepto de Conflicto Interdepartamental se redujo de siete a sólo dos ítems, logrando medidas aceptadas de fiabilidad del instrumento.

TABLA I. COEFICIENTES DEL ALPHA DE CRONBACH POR DIMENSIÓN

\begin{tabular}{|c|c|c|c|c|}
\hline \multirow[t]{2}{*}{ Dimensiones } & \multicolumn{2}{|c|}{$\begin{array}{c}\text { № de ítems de la } \\
\text { escala }\end{array}$} & \multirow{2}{*}{$\begin{array}{c}\text { Alpha } \\
\text { de Cronbach } \\
\text { Inicial }\end{array}$} & \multirow{2}{*}{$\begin{array}{c}\text { Alfa de } \\
\text { Cronbach } \\
\text { si el ítem es } \\
\text { eliminado }\end{array}$} \\
\hline & Inicial & Final & & \\
\hline Análisis de Clientes ac & 9 & 9 & 0.887 & 0.887 \\
\hline $\begin{array}{l}\text { Acciones Estratégicas } \\
\text { sobre los Clientes aec }\end{array}$ & 11 & 11 & 0.930 & 0.930 \\
\hline Análisis de la Competencia aco & 5 & 5 & 0.953 & 0.953 \\
\hline $\begin{array}{l}\text { Acciones Estratégicas sobre } \\
\text { la Competencia aeco }\end{array}$ & 4 & 4 & 0.895 & 0.895 \\
\hline Análisis del Entorno ae & 3 & 3 & 0.876 & 0.876 \\
\hline $\begin{array}{l}\text { Acciones Estratégicas sobre el } \\
\text { Entrono aee }\end{array}$ & 3 & 2 & 0.549 & 0.549 \\
\hline Coordinación Interfuncional cri & 6 & 6 & 0.904 & 0.904 \\
\hline Conflicto Interdepartamental cfi & 7 & 2 & 0.464 & 0.710 \\
\hline Conexión Interdepartamental cni & 6 & 6 & 0.605 & 0.605 \\
\hline Formalización f & 7 & 6 & 0.541 & 0.615 \\
\hline Centralización c & 5 & 5 & 0.865 & 0.865 \\
\hline $\begin{array}{l}\text { Orientación del Sistema de } \\
\text { Recompensas osr }\end{array}$ & 4 & 4 & 0.617 & 0.617 \\
\hline Compromiso Organizacional co & 3 & 2 & -0.467 & 0.453 \\
\hline Espíritu de Equipo ee & 7 & 6 & 0.496 & 0.706 \\
\hline Desempeño Total dt & 2 & 2 & 0.970 & 0.970 \\
\hline Total & 82 & 82 & 0.936 & 0.936 \\
\hline
\end{tabular}

Fuente: Elaboración propia.

\footnotetext{
${ }^{3}$ La prueba alfa de Cronbach se utiliza para el coeficiente fiabilidad que valora la consistencia interna interna (Hair, Anderson, Tatham y Black, 2005).

${ }^{4}$ Las mediciones de consistencia interna evalúan el grado en que los diferentes reactivos miden la misma capacidad, rasgo o atributo (Kaplan y Saccuzzo, 2006).
} 
Para el caso de los efectos de la Orientación al Mercado, se encontró que la dimensión Compromiso Organizacional tiene un Alpha de Cronbach de 0.453, después de haberse hecho el proceso de depuración de ítems, por lo que no pudo ser usada la escala para medir la dimensión en Tabasco. En el caso de las dimensiones Formalización y Espíritu de Equipo, se eliminó un ítem con el proceso de depuración; para el caso de conflicto Interdepartamental se eliminaron cinco de ellos con la finalidad de lograr alcanzar un Alpha de Cronbach mayor de $0.60^{5}$. La tabla I muestra los resultados para cada una de las dimensiones evaluadas.

\section{RESULTADOS}

La información que arrojan los 23 cuestionarios aplicados nos señalan los siguientes resultados:

Con relación al perfil del ejecutivo el $52 \%$ de ellos son presididos por un Director General y el $48 \%$ por Gerentes Administrativos. De ellos, $21.73 \%$ son mujeres y el $78.26 \%$ hombres. En relación a la edad, el $26 \%$ tiene entre 25 y 35 años, el $30 \%$ de 36 a 45 años y el $44 \%$ más de 45 años. El $91 \%$ tiene un nivel de estudios superiores y la antigüedad en su puesto el $52 \%$ tiene 11 años o más.
La calificación más alta de los Índices de Orientación al Mercado la alcanzó el Índice de Análisis del Entorno de manera total, lo que nos muestra la preocupación que tienen los directivos por conocer permanentemente el comportamiento de las variables externas que impactan en el desarrollo de sus actividades pero no están interesados en generar estrategias para preservar el medio ambiente y la calificación más baja la obtuvo el Índice de Análisis de la Competencia, lo que muestra un menor interés de los directivos por conocer la forma de cómo actúan sus competidores y las características de los productos/servicios que ofrecen.

En cuanto a los indices correspondientes a las dimensiones de antecedentes (causas) de la Orientación al Mercado, el índice con la más alta calificación fue de Conexión Interdepartamental, el cual muestra un alto grado de interrelación entre los distintos departamentos que integran las clínicas y hospitales, así mismo podría ser resultado del buen clima organizacional que existe en ellos. La calificación más baja correspondió al Índice de Conflicto Interdepartamental, lo que podría estar confirmando las buenas relaciones que hay entre las personas de los distintos departamentos que integran la empresa.
TABLA II. ÍNDICES DE LOS CONSTRUCTOS Y DIMENSIONES DE LA ORIENTACIÓN AL MERCADO DE HOSPITALES

\begin{tabular}{lcc}
\hline \multicolumn{1}{c}{ Constructor y Dimensiones OM } & \multicolumn{2}{c}{ Media } \\
\cline { 2 - 3 } & Tabasco & Aguascalientes \\
\hline Índice Análisis de Clientes Indac & 5.15 & 4.95 \\
Índice Acciones Estratégicas Con Clientes Indaec & 5.13 & 5.35 \\
Índice Orientación al Cliente Indocl & 5.14 & 5.17 \\
Índice Análisis de la Competencia Indaco & 4.37 & 4.68 \\
Índice Acciones Estratégicas Sobre & 4.63 & 4.91 \\
Competencia Indaeco & & \\
Índice Orientación a la Competencia Indoco & 4.50 & 4.78 \\
Índice de Análisis del Entorno Indae & 5.17 & 4.60 \\
Índice de Acciones Estratégicas sobre el Entorno &.-- & 4.14 \\
Indaee & & \\
Índice de Orientación al Entorno Indoe & 5.17 & 4.37 \\
Índice de Coordinación Interfuncional Indcri & 4.83 & 4.64 \\
Índice de Orientación al Mercado & 4.91 & 4.89 \\
\hline
\end{tabular}

Encuantoalasvariablesconsideradas para el estudio como efectos de la Orientación al Mercado (OM), resultó que en Tabasco el efecto de Compromiso Organizacional no pudo ser evaluado al no presentar consistencia interna. EI Índice de Desempeño obtuvo la mayor calificación lo que muestra que los directivos o gerentes se evalúan con un buen desempeño total: en el año anterior y que sus competidores más importantes; mientras que la calificación más baja corresponde al Índice de Espíritu de Equipo, lo que podría traducirse que en los hospitales y clínicas no existe un sentido de grupo y que se trabaja en forma individual.

Fuente: Elaboración propia.

\footnotetext{
${ }^{5}$ El acuerdo generalmente acepto sobre el límite inferior para alfa de Cronbach es de 0.70 , aunque puede bajar a 0.60 en la investigación exploratorias, tipo de investigación a la que alude esta trabajo. (Hair, Anderson, Tatham y Black, 2005).
} 
TABLA III. ÍNDICES DIMENSIONES DE ORIENTACIÓN AL MERCADO (OM) DE HOSPITALES

\begin{tabular}{lcc}
\hline Constructos y Dimensiones OM & \multicolumn{2}{c}{ Media } \\
\cline { 2 - 3 } Índice Orientación al Cliente Indocl & Tabasco & Aguascalientes \\
Índice Orientación a la Competencia Indoco & 5.14 & 5.17 \\
Índice De Orientación al Entorno Indoe & 5.50 & 4.78 \\
Índice De Coordinación Interfuncional Indcri & 4.83 & 4.37 \\
Índice de Orientación al Mercado & 4.91 & 4.64 \\
\hline
\end{tabular}

Fuente: Elaboración propia.

TABLA IV. ÍNDICES DE LAS DIMENSIONES ANTECEDENTES (CAUSAS) DE LA ORIENTACIÓN AL MERCADO (OM) DE HOSPITALES

\begin{tabular}{lcc}
\hline \multicolumn{1}{c}{ DIMENSIONES ANTECEDENTES DE LA OM } & \multicolumn{2}{c}{ Media } \\
\cline { 2 - 3 } & Tabasco & Aguascalientes \\
Índice de Conflicto Interdepartamental indcfi & 3.04 & 3.14 \\
Índice de Conexión Interdepartamental indcni & 5.33 & 5.33 \\
Índice de Formalización indf & 3.64 & 4.61 \\
Índice de Centralización indc & 3.23 & 3.44 \\
Índice de Orientación al Sistema de & 4.11 & 4.16 \\
Recompensas indosr & & \\
Fuente: Elaboración propia. &
\end{tabular}

\section{DISCUSIÓN}

\section{COMPARACIÓN DE LOS ÍNDICES DE TABASCOY} AGUASCALIENTES

La información obtenida para los hospitales y clínicas del Estado de Tabasco, se comparó con los resultados de la investigación intitulada: «La orientación al Mercado (OM) y su influencia en el desempeño de hoteles y hospitales deAguascalientes», cuyos resultados fueron presentados por sus autoras en la IV Cátedra «Agustín Reyes Ponce» del CUMEX'.

Los índices de los constructos y dimensiones del modelo de Orientación al Mercado de Tabasco yAguascalientes, expresados por los valores de las medias, que se obtienen a través de la utilización del paquete SPSS, se presentan en la tabla II.

Cabe hacer notar que las medias de los Índices de

\footnotetext{
${ }^{6}$ En la mesa \#1, denominada Comportamiento y Cultura Organizacional, celebrado en la Ciudad de Aguascalientes, en junio del 2009. (http://www.cudi.edu.mx/eventos/2009/09_06_25_ CUMEX_uaa.pdf).
}

Orientación a Mercado (Ver Tabla III)entre los hospitales en Tabasco y Aguascalientes son muy cercanas en cuatro dimensiones, observándose una diferencia en el índice de Orientación al Entorno.

Al comparar los Índices correspondientes a los antecedentes (causas) de la Orientación a Mercado (Tabla IV), se observa una diferencia significativa entre los Hospitales de Aguascalientes y Tabasco solo en el Índice de Formalización.

En cuanto a las variables que fueron consideradas para el estudio como efectos de la Orientación a Mercado (OM) (Tabla V), que en Aguascalientes el índice de Compromiso Organizacional tuvo la calificación intermedia, en cambio en Tabasco no pudo ser evaluado con la escala propuesta. Al igual que en Aguascalientes, el Índice de Desempeño Total y el Índice de Espíritu de Equipo presentan el mismo comportamiento.

\section{CORRELACIÓN DE LOS ANTECEDENTES O CAUSAS RELACIONADAS CON LA ORIENTACIÓN AL MERCADO}

Se pude apreciar que las correlaciones son débiles entre el índice de orientación al mercado y los índices 
TABLA V. ÍNDICES DE LAS DIMENSIONES EFECTOS DE LA ORIENTACIÓN A MERCADO (OM) DE HOSPITALES

\begin{tabular}{lcc}
\hline \multicolumn{1}{c}{ Dimensiones efectos de la OM } & \multicolumn{2}{c}{ Media } \\
\cline { 2 - 3 } & Tabasco & Aguascalientes \\
Índice de Compromiso Organizacional indco &.-- & 4.75 \\
Índice de Espíritu de Equipo indee & 4.64 & 4.50 \\
Índice de Desempeño Total inddt & 5.59 & 5.54 \\
\hline
\end{tabular}

Fuente: Elaboración propia.

que miden la Dinámica Interdepartamental y el Sistema Organizacional (antecedentes), en el caso de los Hospitales de Tabasco. En el caso del Conflicto Interdepartamental (-0.15) y el Índice de centralización $(-0.091)$ son negativas, es decir, si disminuye la centralización y el conflicto departamental aumenta el índice de orientación al mercado y si hay un aumento en el índice de conexión interdepartamental, el índice de formalización y el Índice de Orientación del Sistema de Recompensas, aumenta el índice de OM.

Se puede valorar que las correlaciones son débiles entre los efectos y el índice de orientación al mercado, al observarse una correlación negativa del Índice de Espíritu de Equipo (-0.113) con relación al Índice de Orientación al Mercado. En cambio existe un correlación positiva, aunque débil, del $42.8 \%$. Lo que implica que a mayor Orientación del Mercado mayor Desempeño Total.

\section{GRADO DE ORIENTACIÓN AL MERCADO}

Con la finalidad de poder comparar los resultados de ambos estudios se utilizaron la tabla elaborada por Pinzón y Serra (2009) quienes establecieron tres niveles de Orientación al Mercado(OM) con base en los promedios obtenidos para todos los casos, de modo que se consideran un Nivel Bajo de Orientación a Mercado a las unidades de negocios que se ubican entre los rangos 1.00 y 3.00 ; el segundo rango contempla las unidades de negocios que se encuentran entre 3.01 y 5.00 , considerándose que tienen un Nivel Medio de Orientación a Mercado y por último, se estableció un tercer rango que está comprendido por las unidades de negocios que se ubican entre 5.01 y 7.00 , lo que representa a las organizaciones que tienen un Nivel Alto de Orientación a Mercado.

Al hacer una comparación de las frecuencias por rango para cada uno de los Estados estudiados (tabla VI) podemos observar que el mayor porcentaje de hospitales del municipio del Centro del Estado de Tabasco se encuentra en el Nivel Alto de OM (52.20\%). Solamente un $8.70 \%$ de ellos (2) se encuentra en el Nivel Bajo. En Aguascalientes hay mayor cantidad de unidades (hospitales) en el nivel alto, representando el $58 \%$; pero también tienen un $21 \%$ de hospitales en el nivel bajo.

TABLA VI. ANÁLISIS COMPARATIVODEEMPRESAS DE TABASCOYAGUASCALIENTES POR NIVEL DE OM

\begin{tabular}{|c|c|c|c|c|c|}
\hline \multirow[b]{2}{*}{ Niveles OM } & \multirow[b]{2}{*}{ Rango } & \multicolumn{2}{|c|}{ Tabasco } & \multicolumn{2}{|c|}{ Aguascalientes } \\
\hline & & Frecuencia & $\begin{array}{c}\text { Porcentaje } \\
(\%)\end{array}$ & Frecuencia & $\begin{array}{c}\text { Porcentaje } \\
(\%)\end{array}$ \\
\hline Nivel Bajo de OM & $1.00-3.00$ & 2 & $8.70 \%$ & 5 & $21 \%$ \\
\hline Nivel Medio de OM & $3.01-5.00$ & 9 & $39.10 \%$ & 5 & $21 \%$ \\
\hline Nivel Alto de OM & $5.01-7.00$ & 12 & $52.20 \%$ & 14 & $58 \%$ \\
\hline Total & & 23 & $100.00 \%$ & 24 & $100 \%$ \\
\hline
\end{tabular}

Fuente: Elaboración propia. 


\section{CONCLUSIÓN}

Las empresas que buscan permanecer en el mercado deben constantemente renovarse: replanteándose su Visión, Misión y Objetivos; adaptando su estructura y procesos a las características locales, nacionales e internacionales; modificando sus formas de gestión y generando nuevas estrategias que respondan a los cambios económico, político y social de su entorno. Esto implica modificaciones a la cultura organizacional, que generen los comportamientos requeridos para cubrir las necesidades del mercado y procesos de adaptación para un crecimiento interno permanente. Este proceso de inteligencia de mercados se le conoce como la Orientación al Mercado o nueva filosofía del Marketing. El compromiso de los directivos por seguir esta práctica es fundamentalmente para propiciar los cambios, pero muchos gerentes de los sectores industriales, comerciales y de servicios de nuestro país aún no integran esta tecnología a sus empresas, por lo que en los últimos años han perdido competitividad y otras han desaparecido.

Como se establece en el trabajo, en Tabasco el valor encontrado del índice de Orientación al Mercado en los hospitales y clínicas, indica que los gerentes tienen una mayor orientación hacia el análisis del entorno y del cliente que para la competencia; en cambio en Aguascalientes están mayormente centrados en el Cliente. Además mientras en Aguascalientes se determinó el Compromiso Organizacional de los empleados a través de la percepción de los directivos, en Tabasco la escala no permitió determinar el efecto que tiene la OM sobre el compromiso organizacional de los empleados.

La comparación entre los resultados obtenidos de las empresas estudiadas en cada uno de los Estados nos muestra las diferencias en que los directivos de las empresas adoptan las nuevas filosofías y al mismo tiempo la forma de cómo las organizaciones enfrentan los cambios que se generan en el entorno.

Al hacer una comparación de los datos podríamos concluir que la mayor parte de las empresas del sector servicio (hospitales y clínicas) de los dos estados tiene un alto grado de Orientación al Mercado, aunque el Estado de Aguascalientes cuentan con un mayor número de empresas con un grado de $\mathrm{OM}$ bajo.

Se proponen una serie de estrategias en cada uno de los rubros del modelo, las cuales las empresas deberán implementar, dependiendo de las debilidades individúales.

\section{ANÁLISIS DE CLIENTES}

- Desarrollar un esquema que permita medir el grado de satisfacción de los clientes.

- Desarrollar procedimientos que permiten seguir la evolución de las necesidades actuales de los clientes.

- Desarrollar un sistema que les permita conocer los problemas que los clientes pueden tener con los productos /servicios de la empresa.

- Realizar estudios para analizar las ventajas e inconvenientes que tienen la entidad de formas nuevas y alternativas de venta y atención al cliente (cajeros automáticos, contacto telefónico y vía informática).

\section{ACCIONES ESTRATEGICAS DE CLIENTES}

- Identificar los tipos de soluciones que se brindan a los clientes y verificar si están muy bien adaptadas a sus necesidades y no sean simplemente productos y servicios estándares.

- Estudiar los conceptos de los productos/servicios que responderán a las necesidades futuras del mercado.

- Comercializar sistemáticamente productos/servicios innovadores.

- Identificar la velocidad que tienen para responder a los cambios en las necesidades de los clientes y a las quejas formuladas por ellos.

- Desarrollar un plan de marketing que coordina acciones sobre los productos/servicios, la venta, la comunicación y el precio o costo para el cliente.

- Brindar información completa a clientes con el objeto de que utilicen y aprovechen plenamente los productos/servicios y se encuentren totalmente satisfechos con ellos.

\section{ANÁLISIS DE LA COMPETENCIA}

- Conocer muy bien los objetivos y estrategias de los competidores más peligrosos.

- Disponer de un sistema que permita un buen conocimiento de los puntos fuertes y débiles de los competidores más peligrosos.

- Contar con un sistema para el seguimiento preciso de la evolución de la política de marketing de los competidores más peligrosos.

- Detectar las amenazas que representan los productos/servicios sustitutos.

- Conocer bien las características técnicas y la imagen de los productos/servicios de la competencia. 
ACCIONES ESTRATÉGICAS SOBRE LA COMPETENCIA

- Desarrollar un esquema que permita responder rápidamente a las acciones más peligrosas de la competencia.

- Emprender acciones sistemáticas para anticiparse y sorprender a la competencia.

- Adoptar un comportamiento competitivo bien definido frente a sus competidores más peligrosos.

- Diferenciar sus productos/servicios de los competidores en características importantes para el cliente

\section{ANÁLISIS DEL ENTORNO}

- Desarrollar sistemas que permiten seguir de cerca los impactos en el entorno legal, tecnológico, económico, etc.

- Identificar los factores sensibles que pueden tener un impacto en el negocio.

- Elaborar indicadores previos que les permita vigilar los factores sensibles y los factores de riesgo.

\section{ACCIONES ESTRATEGICAS SOBRE EL ENTORNO}

- Elaboración de un plan estratégico, utilizamos el método de los escenarios y definir una o varias estrategias alternativas.

- Desarrollar estrategias que consideran el medio ambiente y la defensa del entorno en general como un elemento de las mismas.

\section{COORDINACIÓN INTERFUNCIONAL}

- La información importante sobre el mercado siempre debe ser difundida a todas las áreas funcionales de la empresa.

- Las estrategias de marketing deben ser elaboradas concertadamente con las otras funciones o áreas de la empresa.

- Implantar acciones para que cada individuo de la empresa se sienta personalmente al servicio del mercado.

- Organizar reuniones interfuncionales para analizar toda la información importante recabada sobre el mercado.

- Estimular los intercambios de información entre las diferentes funciones de la empresa.

- Implantar procedimientos para que cada función o área reciba información sobre su contribución a la satisfacción de los clientes.

\section{CONFLICTO INTERDEPARTAMENTAL}

- Desarrollar esquemas para que todos los departamentos de la organización lleven una adecuada relación de trabajo.
- Comunicar la compatibilidad de los objetivos entre los distintos departamentos de la organización.

\section{CONEXIÓN INTERDEPARTAMENTAL}

- Desarrollar un esquema de comunicación que fluya de abajo hacia arriba y viceversa, que asegure la charla entre los diferentes miembros de la organización.

\section{FORMALIZACIÓN}

- Desarrollar un conjunto de reglas flexibles que permita al empleado tomar decisiones que beneficien el crecimiento de la organización y de los empleados.

\section{CENTRALIZACIÓN}

- Establecer esquemas que eviten una centralización total en la toma de decisiones.

ORIENTACIÓN DEL SISTEMA DE RECOMPENSAS

- Desarrollar un esquema de recompensas que motiven al empleado a prestar un mejor servicio.

\section{COMPROMISO ORGANIZACIONAL}

- Diseñar un esquema que permita motivar al empleado a generar un compromiso con la organización.

\section{ESPIRITU DE EQUIPO}

- Diseñar un esquema que permita al empleado generar un espíritu de equipo.

Finalmente podemos señalar a partir de los datos obtenidos en el estudio que el modelo de Orientación al Mercado muestra una viabilidad de aplicación a las empresas mexicanas, medianas y grandes, por lo menos en lo que se refiere al sector servicios y específicamente a la rama de Hospitales y clínicas. Lo que nos lleva a realizar futuras investigaciones con otros sectores con el fin de verificar si el modelo puede ser aplicado a todos los sectores de la economía. Es importante señalar que este tipo de estudios nos permite contar con una herramienta que debe socializarse en el aula con el fin que el estudiante aprenda a aplicar la teoría a la realidad y a la vez de ésta pueda obtener la información necesaria que lo lleve a generar sus propios modelos. 
Anderson, D., Sweeney D. y Williams, T. (2004). Estadística para administración yeconomía. México: Thomson.

Bigné, E., Küster, I., Andreu, B., yAndreu, L. (2001). La medición de la orientación al mercado en los sectores cerámico y turístico de la comunidad valenciana: propuesta y validación de una escala a medida. Proyecto de investigación. Instituto Valenciano de Investigaciones Económicas, S. A.

Cea Dàncona, Ma. (2001). Metodología cualitativa: estrategias y técnicas de investigación social. Madrid, España: Síntesis Sociología.

Hair, Joseph F. Jr., Anderson Rolph E., Tatham Ronald L. y Black William C. (2005). Análisis Multivariante (5a. ed.). España: Person/Prentice Hall.

Iguaran, R., Chávez, E., y Pérez, J. (2006). Satisfacción de los clientes del servicio médico odontológico de la Universidad de Zulia. Núcleo Costa Oriental del Lago. Revista Venezolana de Ciencias Sociales. Universidad Nacional Experimental Rafael María Baralt, Unermb. Venezuela.

Kohli, Ajai y Kaworski, Bernard J. (1990). Market orientation: the construct, research proposition and managerial implications. Journal of Marketing, vol. 54, U.S., 1990.

Kotler, P. (1981). Dirección de Mercadotecnia: análisis, planeación y control. México: Diana.

Lonchi i Andreu, J. (1993). La orientación al mercado y sus efectos en los resultados de la empresa (Tesis doctoral). Universidad Autónoma de Barcelona, España.

Lonchi i Andreu, J.; López Belbeze Ma., y Eusebio, R. (1999). Orientación al aprendizaje, orientación al mercado, resultados y el efecto moderador de la procedencia funcional del Director General. Barcelona, España.
Narver, C., y Slater , F. (1990). The effect of a market orientation on business profitability. Journal of Marketing, U.S.

Olavarrieta, S., Friedmann, R., Hidalgo, C. (1999). La orientación al mercado de una nación en desarrollo: Un estudio de validación con empresas Chilenas. Revista Científica de America Latina y el Caribe, España y Portugal. Universidad Autónoma del Estado de México.

Pérez, T. H. (1987). Estadística para ciencias del comportamiento. México: Harla.

Pinzón, S. (2009). Impacto de la Orientación a Mercado en la Innovación en empresas de Aguascalientes (Tesis doctoral). Universidad Autónoma de Aguascalientes.

Pinzón, S. y Martínez, M. (2009). La orientación al mercado (OM) y su influencia en el desempeño en hoteles y hospitales de Aguascalientes. Investigación del Postgrado. UniversidadAutónoma de Aguascalientes.

Riveros, J. y Berné, C. (2006). La aplicación del marketing en hospitales públicos desde la perspectiva de los funcionarios: El caso de un hospital del sur de Chile. Rev Méd Chile.

Santos, Ma., Vázquez, R., y Álvarez, L. (2002). La orientación al mercado como fuente de ventajas competitivas: antecedentes asociados al equipo directivo y a la estructura organizativa en las empresas industriales. Revista Asturiana de Economía.

Scheaffer, R.; Mendenhall, W. y Otto, L. (1981). Elementos de muestreo (G. Rendón y J. R. Gómez, Trads.). México: Grupo Editorial Iberoamérica (Trabajo original publicado en 1971).

Sierra, R. (2007). Técnicas de investigación social: teoría y ejercicios. México: Thomson.

SPSS Inc. (2007). Statistical package for the social sciences (versión 15.0) [Sofware de cómputo]. 
Chicago, Illinois, EE. UU.

Valenzuela, L., García de Madagariaga, J., y Blasco, Ma. (2006). Evolución del marketing hacia la gestión orientada al valor del cliente: revisión y análisis. Teoría, Ciencia. Arte y Humanidades. Universidad del Bío- Bío. Chile. Red de Revistas Científicas de América Latina y el Caribe, España y Portugal. Universidad Autónoma del Estado de México.

Valenzuela, L., García de Madagariaga, J., Blasco Ma. (2007). Orientación al valor del cliente y las nuevas métricas de marketing. Revisión y análisis. Panorama Socioeconómico. Universidad de Talca. Chile. Red de Revistas Científicas de América Latina y el Caribe, España y Portugal. Universidad Autónoma del Estado de México.

Velasco, H., y Díaz de Rada. (2003). La lógica de la Investigación Etnográfica: Un modelo de trabajo para etnógrafos de escuela. Madrid, España: Trotta, S.A.

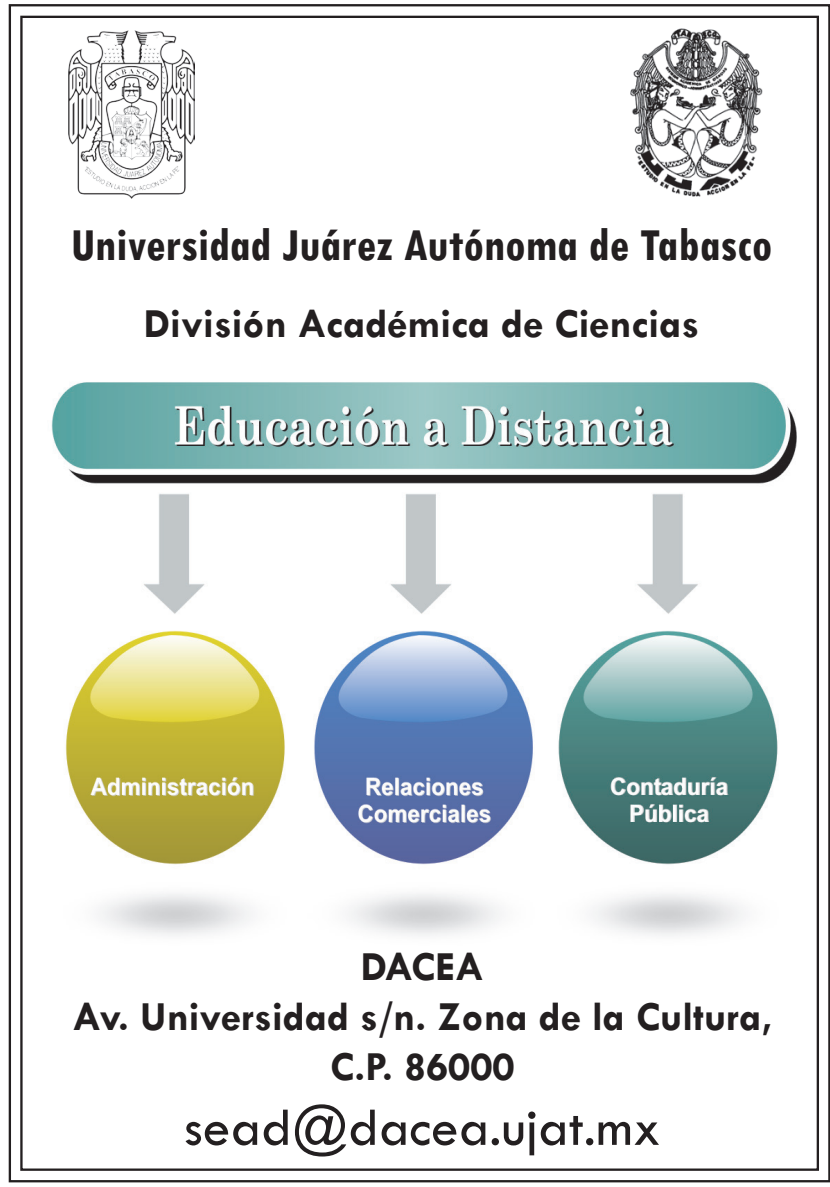

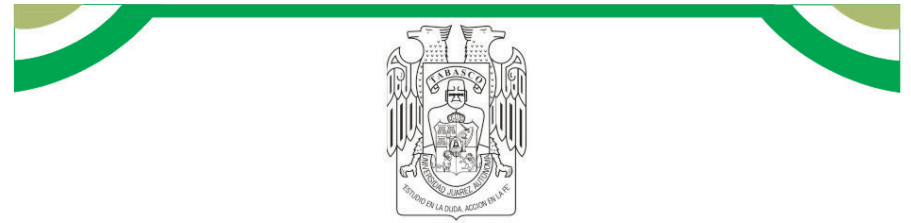

Universidad Juárez Autónoma de Tabasco

División Académica de Ciencias Económico Administrativas

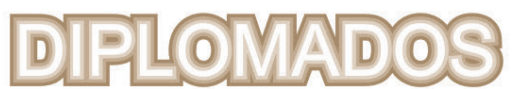

Desarrollo del factor humano en la administración

\section{Mercadotecnia de servicios}

\section{Contribuciones fiscales}

\section{Desarrollo de habilidades administrativas}

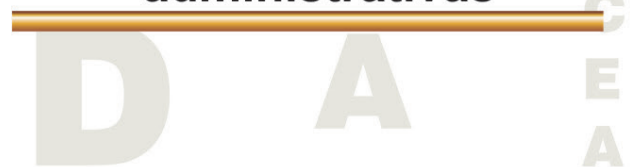

Con el objetivo de contribuir a la actualización profesional, la División Académica de Ciencias Económico Administrativas, ha desarrollado programas de diplomados y cursos de alta calidad, que promuevan la capacitación continua dentro de las instituciones públicas y privadas.

\section{Informes:}

Edificio de Educación Continua y a Distancia.

Av. Universidad Zona de la Cultura, Villahermosa, Tabasco.

\section{M.A. William Baldemar López Rodríguez}

Tels: 3 -58-15-00 Ext. 6219

E-mail: educacioncontinua.dacea@ujat.mx 